Research Article

\title{
Compatibility of Composite Biomaterials in Sports Injury Repair
}

\begin{abstract}
Mo Xing (1D)
School of Physical Education, Hulunbuir University, Hulunbuir 021000, Inner Mongolia, China

Correspondence should be addressed to Mo Xing; xingmo@hlbec.edu.cn

Received 19 May 2021; Revised 27 July 2021; Accepted 11 August 2021; Published 20 August 2021

Academic Editor: Song Jiang

Copyright (c) 2021 Mo Xing. This is an open access article distributed under the Creative Commons Attribution License, which permits unrestricted use, distribution, and reproduction in any medium, provided the original work is properly cited.

With the rapid development of sports, sports injuries have become an inevitable problem in sports. Repairing sports injuries has always been the focus of attention in the field of sports and medicine. The wide application of biomedical materials leads to the innovation and development of medical technology. It is of great significance to use them to repair sports injuries, but the current research results are not ideal. In this paper, the biocompatibility of composite biomaterials used to repair sports injuries was studied. First, the rat bone marrow mesenchymal stem cells were cultured to prepare a cell suspension. Then, the tricalcium phosphate-gelatin composite scaffold material and the collagen material were scraped into powder to make the material extract, which are, respectively, the experimental group and the control group. An apoptosis detection kit and a flow cytometer were used to detect apoptosis rate and survival rate of all samples, and a CKK-8 kit was used to detect cytotoxicity and calculate cell proliferation activity. The absorbance value was read with a microplate reader, and the coagulation performance and hemolysis rate of different groups were calculated. The experimental results showed that the cell survival rate of the experimental group was $91.41 \%$, the early apoptosis rate was $2.81 \%$, the stem cell proliferation activity was 0.83 , and the anticoagulant performance and hemolysis rate were $64.8 \%$ and $0.28 \%$, respectively. This indicates that although the anticoagulant performance of the tricalcium phosphate-gelatin composite scaffold is weak, its cell proliferation activity is strong, which is more suitable for cell adhesion and proliferation, and the cell apoptosis and hemolysis rate are lower. Therefore, it has better biocompatibility than a single collagen material.
\end{abstract}

\section{Introduction}

1.1. Background Significance. Sports injury not only endangers the health of athletes, but also has an irreversible impact on their life and sports career. Therefore, it is necessary not only to prevent sports injury in advance, but also to take scientific and effective repair methods after the injury occurs [1]. When sports injury is serious, it is necessary to implant biomaterials to replace damaged tissues or organs for repair. At present, many biomaterials have been used to repair sports injury [2]. Especially for the composite biomaterials which combine the advantages of a variety of materials, it is necessary to study the biocompatibility of these new composite materials, so as to ensure that there will be no new damage to the human body after implantation. Bioremediation materials include the following categories: (1) biomedical metal materials: medical metal materials are metals or alloys used as biomedical materials, which have high mechanical strength and fatigue resistance, and are the most widely used clinically for load-bearing implants. The materials mainly include artificial joints and artificial bones made of cobalt alloy (Co-Cr-Ni), titanium alloy (Ti-6Al-4V), and stainless steel. Nitinol shape memory alloy has the intelligent characteristics of shape memory and can be used in orthopedics and cardiovascular surgery. (2) Biomedical polymer materials: there are two types of biomedical polymer materials: natural and synthetic. The fastest growing is synthetic polymer medical materials. Through molecular design, many biomaterials with good physical and mechanical properties and biocompatibility can be obtained. Among them, soft materials are often used as substitutes for human soft tissues such as blood vessels, esophagus, and knuckles; synthetic hard materials can be used as artificial dura mater, spherical valves of cage-shaped artificial heart valves, etc.; liquid synthetic materials such as room temperature vulcanization silicone rubber can be used as an 
injectable tissue repair material. (3) Biomedical composite materials: biomedical composite materials are biomedical materials composed of two or more different materials. They are mainly used to repair or replace human tissues and organs or enhance their functions and the manufacture of artificial organs. Among them, titanium alloy and polyethylene tissue prostheses are often used as joint materials; carbon-titanium synthetic materials are good artificial femoral heads for clinical applications; polymer materials and biopolymers (such as enzymes, antibodies, antibodies, and hormones) can be combined as a biosensor.

1.2. Related Work. The repair of sports injuries has always been the focus of the sports world. Scillia et al. proposed a surgical technique including rectus abdominis repair and long adductor muscle lengthening, aimed at patients with sports muscle injuries that are incurable by conservative treatment [3]. Their research only focused on muscle injuries. In fact, many sports injuries can cause bone tissue damage. Composite biomaterials have attracted the attention of researchers from various countries due to their unique properties. Wang et al. reviewed the application of nano-hydroxyapatite and its composite biomaterials in bone defect repair. They discussed whether the application of composite materials and polymer biomaterials can overcome the brittleness and poor mechanical properties of nano-hydroxyapatite [4]. Pieniak et al. discussed the quantitative fatigue assessment of polymer-ceramic composites for dental restorations and simulated thermal fatigue using a dedicated computer control device with thermal cycling algorithm [5]. Li et al. prepared a bio-scaffold material by melt fusion/particle leaching process and analyzed the biocompatibility of the material [6]. Their experiments provide a reference for the research of composite biomaterials, but when conducting research, they either tend to explore the physical properties of the materials, or prefer biocompatibility without combining the two.

1.3. Innovative Points in This Paper. This paper studies the compatibility of composite biomaterials in sports injury repair, combined with the role of biomaterials in injury repair; this paper innovatively uses flow cytometry to detect the cells of tricalcium phosphate-gelatin composite scaffolds and collagen materials. In observing cell proliferation activity, this article selects the CKK- 8 kit, which is more suitable as an environment for cell proliferation than traditional kits.

\section{Sports Injury Repair and Compatibility of Composite Biomaterials}

\subsection{Sports Injury Repair}

2.1.1. Cause of Damage. Different sports will cause different parts of the injury. Common sports injuries include periostitis, fractures, muscle strains, and ankle sprains. Injuries that occur during sports activities can be divided into acute injuries and chronic injuries [7]. Acute injuries happen suddenly, and if improper treatment at the time leads to recurrence of later illnesses, it will turn into chronic injuries.

The main cause of sports injuries is technical errors or irregularities during the exercise. Professional athletes also make mistakes in their movements, let alone ordinary people. Their exercise time is not long, and they cannot master some difficult movements. These wrong movements violate the physiological characteristics and biomechanical principles of the human body and can easily lead to sports injuries. In addition, improper preparation activities and failure to allow the body to adapt to exercise will increase the probability of muscle strain and joint sprain. Exercise load exceeds the level that the body can withstand, which can easily lead to body fatigue and minor injuries. If it is not repaired in time, it will induce serious sports injuries [8].

After a sports injury occurs, it is also important to take a reasonable first aid plan to minimize the degree of sports injury. Some sports coaches have insufficient training experience, fail to reasonably convey the essentials of sports movements, or do not arrange training content according to the actual situation, which will lead to sports injuries.

Sports venues and equipment facilities will also affect the formation of sports injuries. The geology of the site is excellent, the cushioning performance is poor, and the equipment is outdated, which cannot guarantee the safety of the athletes and bring safety risks [9].

2.1.2. Damage Prevention. There are different preventive measures for damage caused by different reasons. Starting from the athletes themselves, improve the athletes' ideological understanding, publicize basic sports knowledge, and make them aware of the importance of preventing sports injuries. Do adequate preparation activities, which is often said to warm up before exercise to improve the excitability of the human nervous system. Appropriate relaxation should be carried out after exercise to eliminate fatigue caused by exercise [10].

Sports coaches or physical education teachers must put safety first and arrange training scientifically and reasonably according to the physical fitness of the athletes. The intensity and difficulty of exercise must be well controlled to reduce sports injuries caused by improper training plans or excessive exercise [11]. The coach or teacher himself should strengthen the learning of sports health knowledge and guide the athletes to treat sports injuries correctly.

In terms of sports conditions, it is necessary to strengthen the maintenance of venues and equipment, conduct regular inspections, repair and replace damaged equipment in time, and eliminate safety hazards [12]. Athletes themselves should conduct a general safety inspection of the venue and equipment before exercising, and avoid exercising on unsafe venues.

2.1.3. Damage Repair. There are different repair methods for different parts of the injury. After an acute knee injury occurs, it is necessary to quickly apply ice to find the cause of the injury for further examination and treatment. Rehabilitation training is carried out on the second day after 
injury to prevent muscle atrophy around the injury. In the recovery period of the exercise range, you must first start training the surrounding muscle strength, and you must do it slowly to avoid excessive stimulation. If you experience discomfort after training, you should stop using ice immediately [13]. In the strength recovery phase, attention should be paid to load control and protection during training period, and training equipment with a constant angle and speed should be selected. In the functional recovery period, increase the intensity of training, pay attention to the recovery of the neuromuscular system of the athlete's knee joint, and the methods of strength training should be diversified and flexible.

You also need to apply ice immediately after acute ankle injury to reduce the congestion and swelling of the injured area. At the same time, try to avoid activities and raise the limbs to relieve pain. Those with severe injuries need to be sent to the hospital for examination and treatment immediately, and acupuncture and drug treatment can be performed later, with functional recovery training.

The directional repair process of fatigue damage of bone tissue is completed by bone cells, and the directional bone reconstruction work is achieved by osteoclasts and osteoblasts [14]. There is a threshold effect in the perception and repair of fatigue damage of bone tissue, and bone cells can induce the subsequent directional bone reconstruction process through apoptosis or non-apoptosis. The degree of fatigue damage will directly determine its specific response.

\subsection{Composite Biomaterials in Damage Repair}

2.2.1. Basic Elements of Composite Biomaterials. To be implanted into the human body as a qualified implant, the composite biomaterial must meet the following requirements. First of all, it must have biocompatibility. The basic concept is nontoxic effects. The premise of nontoxic effect is that the chemical structure of the material is stable and the raw material is pure [15]. It will not cause irritation and damage to the tissues, or immune interference and rejection, and will not cause hemolysis or coagulation.

The second is biomechanics, because many biocomposite materials will be used to replace the load-bearing tissues in the human body. If there are not enough mechanical properties, it will easily break. Good mechanical properties include the elasticity, wear resistance, strength, and aging resistance of the material itself, as well as the compatibility with the elastic modulus of the surrounding tissues.

An important sign of biological stability is whether the material can exhibit a good proliferation response phenomenon [16]. After the material is moved into the living body and contact with the recipient tissue, it will form a different structural state after a period of time. The ideal material will have a proliferation reaction phenomenon on the surface, thereby forming a cell layer dominated by fibrocytes. As time goes by, fibrocytes gradually increase, and a good tissue envelope is formed.
Biodegradability requires that the material be slowly degraded after implantation in the organism and replaced by cells, and the rate of degradation and tissue growth must be consistent. Regardless of whether the materials that have been formed in the tissue have not been degraded, or the tissue is degraded before it is completely formed, it is said that it is not conducive to tissue repair. At the same time, some small molecular impurities will inevitably remain in the preparation process of the material. Once it becomes a body fluid exudate, it will cause a serious physiological reaction. Therefore, attention must be paid to its biological exudation $[17,18]$.

The natural fiber used as the raw material of bio-based composite materials includes various types of natural polysaccharides such as cellulose, hemicellulose, tannins, etc. From the physical properties, the surface is hydrophilic, while bio-based composite materials are another large class of raw materials. It is an organic synthetic polymer resin with hydrophobic surface. The surface properties of the two have a huge difference. Due to the weak interface interaction force and easy defects, it is not good for the formation of composite materials. Using these two types of raw materials to produce composite materials can improve the performance of composite materials by modifying fibers and resins to increase the interaction force between the interfaces. However, after the interface performance of the composite material has been improved, the impact and other properties have declined, and the best solution has not been found in a variety of modification treatment methods. From the perspective of biocompatibility, if the use of the material is different, the scale of the evaluation of biocompatibility will be different. For example, in vitro and in vivo (comparison of debridement materials and some materials used for transplantation to help regeneration), debridement materials do not cause inflammation, are nontoxic, and are antibacterial. Regeneration materials may also need to promote cell growth. It still depends on the specific problem. The repair materials used in orthopedics may promote the adhesion and growth of bone cells, and the vascular repair materials must promote the adhesion and growth of endothelial cells, avoid calcification, and also have anticoagulation to avoid thrombosis.

2.2.2. Seed Cells and Growth Factors. Seed cells can help tissue construction and organ regeneration, so they must meet certain criteria to become qualified seed cells. The source of seed cells should be extensive, sufficient in number, and relatively easy to obtain. Seed cells should be easy to cultivate, have strong proliferation, and be expanded in large numbers. Cells with stable genetics and specific biological functions, and with specific functions, should dominate. The immune rejection reaction is minimal or absent, so as to avoid rejection. The molecular structure and function of the seed cell must be similar to that of the normal cell of the regenerated tissue, so as to be better adapted.

At present, seed cells can be divided into stem cells, bone cells, fibroblasts, and endothelial cells [19]. Mesenchymal stem cells have the potential to differentiate into bone and 
adipose tissue. Fibroblasts have the advantages of strong function, rapid proliferation, and low immunogenicity. Endothelial cells can accelerate the process of vascularization and vascular system reconstruction.

Growth factors can combine with specific and high affinity receptors on cell membrane to induce and stimulate cell proliferation and maintain cell survival. The role of growth factors is specific, so it needs to be selected for different cells, tissues, and organs. Fibroblast growth factor can promote angiogenesis and repair. Transforming growth factor can induce cartilage differentiation and promote bone healing and anticancer. Epidermal growth factor can promote cell migration to the wound and accelerate wound healing.

2.2.3. Support Material. The scaffold material provides a platform and support for the growth of seed cells, maintains the original shape and integrity of the tissue, and provides a good environment to promote cell growth, proliferation, and differentiation. The qualified scaffold has a three-dimensional porous structure and simulates the natural microenvironment. The current scaffold materials are divided into three types: natural biological materials, synthetic materials, and composite materials.

Chitosan, collagen, and fibrin are more commonly used in natural biomaterials. These materials have no toxic and side effects, can help cells better adhere to, proliferate, and differentiate, and can also fight infection and promote the recovery of immune function [19]. But it has a certain degree of immunogenicity, and the degradation rate is not very stable. Synthetic materials mainly include polyvinyl alcohol, polystyrene, and polycaprolactone, etc., which have the advantages of many varieties, strong plasticity, and good mechanical properties, and can be mass-produced. However, the biocompatibility and hydrophilicity of these materials are relatively poor, and the degradation products may be toxic and interfere with tissue regeneration. Composite materials combine the excellent properties of different materials to meet different needs. Currently, the composite scaffold materials that have been put into use include chitosan-calcium polyphosphate composite scaffold, tricalcium phosphate-gelatin composite scaffold, and hydroxyapatite polycaprolactone [20].

In order to ensure the safety and effectiveness of scaffold materials during use, physical and chemical performance tests, biological performance tests, and cell and histocompatibility tests must be carried out before clinical use. The physical property test should be carried out in a simulated body fluid environment to test tensile strength, viscoelasticity, hardness, and melting point [21]. The chemical performance test is aimed at $\mathrm{pH}$, hydrophilicity, adsorption, etc. The biological performance test detects the toxicity of the material to the receptor, including cell compatibility and tissue compatibility.

2.3. Evaluation of Biocompatibility. Various biological materials implanted in the body will have different degrees of contact with collective tissues or body fluids, and the reactions caused by contact are divided into two types. One is to prevent reactions, toxic reactions and allergic reactions, local or systemic effects caused by the $\mathrm{pH}$ of the material, residual or adsorbed toxic low-molecular substances, etc. [22]. The second is the reaction of various factors in the body to the deformation, aging, or degradation of the implanted material. Therefore, no matter what kind of biological material, it must meet the requirements of biocompatibility. The methods of evaluating the biocompatibility of biological materials are mainly divided into in vitro cell biology evaluation and in vivo histological evaluation [23]. In vitro cell biology evaluation studies the cell adhesion and growth on the surface of the material, while in vivo histological evaluation observes tissue growth.

2.3.1. Detection Method of Cytotoxicity. A common method for testing biocompatibility is to conduct a cytotoxicity test. Through in vitro cell culture, the effect of biomedical materials on cell growth is detected to evaluate whether the materials are toxic. The use of cytotoxicity testing for biocompatibility evaluation has the advantages of simple operation, rapidity, low cost, and good repeatability.

Cytotoxicity test can be divided into MTT method, agar covering hair, molecular filtration method, cell morphology, and LDH release method according to the standard [24, 25]. The MTT method is generally used to detect the toxicity of the material's eluate. It has the advantages of being simple and accurate and having a high degree of automation. However, the use of the material extract will produce colored precipitates, and fading will occur in a short time. The agar covering method is suitable for large quantities of materials with high toxicity and has the advantages of simple operation and wide application types, but its sensitivity will be affected by the degree of diffusion of the material's eluate on the agar.

The molecular filtration method is suitable for materials with small molecular weights of toxic components. It has the advantage of simultaneously observing the primary and secondary cytotoxicity of the material, but it will forcefully affect the diffusion of the precipitated product in the material. The cell morphology method is suitable for mass testing and has the advantages of simple operation and intuitive results, but the result is an estimate and not very convincing [26]. The $\mathrm{LDH}$ release method is suitable for small batch testing and has the advantages of good objectivity, easy detection, and no labeling process, but it is easily affected by the activity of lactate dehydrogenase and is not easy to control.

\subsubsection{Evaluation Method of Blood Compatibility. Blood} compatibility requires that the material does not produce hemolysis or clots on the surface of the material when it comes into contact with blood, and the material neither destroys the blood components nor changes the blood physiological environment. At present, the blood compatibility of biomaterials is mainly studied through the hemolysis rate, dynamic clotting time, and the number of platelets attached to the surface of the material. 
The blood compatibility of biomaterials is affected by the structure, morphology, composition, and surface free energy of biomaterials [27]. The coagulation properties are also related to the structure of the materials, and also to the contents of platelets, plasma proteins, and coagulation factors in the blood. The hemolysis rate of the material can be determined by detecting the absorbance of the material. After contact with the material, the more hemoglobin released from the blood, the higher the absorbance, the higher the hemolysis rate. The hemolysis rate meeting the biomaterial standard must be lower than 5\%; otherwise the material will have hemolytic effect and cannot be implanted into the body [28].

The blood compatibility of the material can be improved to varying degrees through certain surface treatment methods. The surface of hydrophilic materials has weak interaction with platelets, which will reduce the degree of adhesion and prevent the formation of platelet thrombus.

\section{Experiments on Compatibility Testing of Composite Biomaterials}

\subsection{Experimental Materials, Instruments, and Reagents}

3.1.1. Experimental Materials. In the experimental group, tricalcium phosphate-gelatin composite scaffolds were used in sports injury repair. The control group used collagen, a natural biomaterial. The biomaterials were provided by the hospital without preparation. The experimental animals included 4 clean SD rats, weighing about $210 \mathrm{~g}$. The cells were rat bone marrow mesenchymal stem cells. Rats used in the experiment were also provided by the hospital.

3.1.2. Experimental Equipment. Experimental instruments included multifunctional ultrapure water machine, electronic balance, filter, $\mathrm{CO} 2$ incubator, digital display constant temperature water bath, inverted optical microscope, ultraclean workbench, microplate reader, centrifuge, magnetic stirrer, and micropipette.

3.1.3. Experimental Reagents. They included $75 \%$ alcohol, fetal bovine serum, trypsin, CKK- 8 kit, 4\% paraformaldehyde, penicillin and streptomycin, collagenase, PBS solution, DMEM medium, and apoptosis detection kit.

3.2. Cell Culture. The two rats were killed by severed necks, the thigh bones were removed after disinfection, and the bone marrow mesenchymal stem cells were flushed out from the bone marrow with a syringe. The cells were cultured in DMEM medium, which contained $10 \%$ fetal bovine serum and $100 \mu \mathrm{g} / \mathrm{ml}$ penicillin and streptomycin. They were incubated in a $\mathrm{CO}_{2}$ incubator at $37^{\circ} \mathrm{C}$, and the medium was changed every 2 days. When the number of monolayer cells exceeded $75 \%$, they were digested with trypsin, centrifuged in a centrifuge, and resuspended in culture medium. After the primary cells were obtained, the cells were passaged and the second and third generation cells were obtained for use.

\subsection{Biocompatibility Testing}

3.3.1. Flow Cytometry Detection of Cell Apoptosis Rate. Take the third generation of stem cells in the logarithmic growth phase, and add $1.5 \mathrm{ml}$ trypsin to the culture flask. Put the culture flask in a $\mathrm{CO}_{2}$ incubator at $37^{\circ} \mathrm{C}$ for $3 \mathrm{~min}$. After digestion, pipette the solution with a pipette to become a uniform suspension, centrifuge at $1200 \mathrm{r} / \mathrm{min}$ for $3 \mathrm{~min}$, and remove the supernatant.

The tricalcium phosphate-gelatin composite scaffold material and collagen material were scraped into powder, soaked in $75 \%$ alcohol for 48 hours, then air-dried, and sterilized by ultraviolet radiation. It was made into a material suspension with PBS solution with a concentration of $0.05 \mathrm{~g} /$ $\mathrm{ml}$. Tricalcium phosphate-gelatin composite scaffold material suspension was added to the cell suspension of the experimental group, collagen material was added to the cell suspension of the control group, and PBS solution was added to the cell suspension of the blank group. After culturing for 48 hours, they were washed with PBS solution to remove residual trypsin.

All samples were tested for cell apoptosis rate with a cell apoptosis detection kit and flow cytometer, and the operation process was carried out in accordance with the kit instructions.

3.3.2. Cytotoxicity Test. The treatment of MSCs and materials was the same as that of the other materials. Use CKK-8 kit and operate according to the instructions. But it should be noted that the bubble should be punctured with a hot needle in order to avoid affecting the experimental results. The absorbance values in different sample holes were read by the $450 \mathrm{~nm}$ wavelength of enzyme standard instrument. The calculation method of cell proliferation activity is shown as follows:

$$
\operatorname{RGR}(\%)=\frac{E_{0}-N_{0}}{C_{0}-N_{0}} \times 100 \% .
$$

Among them, $E_{0}, N_{0}$, and $C_{0}$ are the absorbance of experimental group, blank group, and control group.

3.3.3. Evaluation of Blood Compatibility. Take $4 \mathrm{ml}$ of fresh blood from the rat, add it to a centrifuge tube containing anticoagulant, and add physiological saline for dilution. The tricalcium phosphate-gelatin composite scaffold material and the collagen material were scraped into powder, and PBS solution was added after 24 hours of ultraviolet irradiation to prepare a material suspension with a concentration of $0.2 \mathrm{~g} /$ $\mathrm{ml}$, and then filtered. They were also divided into 3 groups, the tricalcium phosphate-gelatin composite scaffold material was the experimental group, the collagen material was the control group, and only the PBS solution was the blank group.

Add $200 \mu \mathrm{l}$ of anticoagulated whole blood to the three groups of material suspensions, and place them in a $37^{\circ} \mathrm{C}$ water bath for 5 minutes. Then start timing and take them out in four time periods of $15 \mathrm{~min}, 30 \mathrm{~min}, 60 \mathrm{~min}$, and $120 \mathrm{~min}$. Centrifuge at a speed of $1200 \mathrm{r} / \mathrm{min}$ for 3 minutes. 
Use the $450 \mathrm{~nm}$ wavelength of the microplate reader to read the absorbance values in different sample wells. The calculation method of the anticoagulant ability of the material is shown as follows:

$$
\mathrm{BCI}=\frac{E_{n}}{C_{n}} \times 100 \% .
$$

Among them, $E_{n}$ and $C_{n}$ represent the absorbance value of the experimental group and the control group, respectively.

The preparation process of the material suspension for the hemolysis test is the same as that of the coagulation test. $1 \mathrm{ml}$ of fresh rat blood is collected and centrifuged at $2500 \mathrm{r} /$ min in 12 minutes, and the supernatant is removed and washed with saline. After obtaining the red blood cells, add $5 \mathrm{ml}$ of normal saline for dilution. Set up the experimental group, the control group, the negative control group, and the blank group, respectively, adding tricalcium phosphategelatin composite scaffold material extract, collagen material extract, physiological saline, and distilled water. There are 3 parallels for each group. After adding the red blood cell suspension in a water bath at a constant temperature for 25 minutes, let it stand for 60 minutes for observation. Centrifuge at a speed of $1800 \mathrm{r} / \mathrm{min}$ for $8 \mathrm{~min}$, take the supernatant, puncture the bubbles with a heating needle, and read the absorbance value at $540 \mathrm{~nm}$ with a microplate reader. The calculation method of the hemolysis rate of the material is shown as follows:

$$
\mathrm{HR}=\frac{E_{1}}{N_{1}} \times 100 \% .
$$

Among them, $E_{1}$ and $N_{1}$ are, respectively, the absorbance values of the experimental group and the blank group.

\section{Discussion on Composite Biomaterial Compatibility}

4.1. Test Results of Apoptosis Rate. Use flow cytometry to observe the apoptosis of experimental group $E$, control group $C$, and blank group N. F1 represents cells that die from debris and damage, F2 represents late-stage apoptotic or necrotic cells, F3 represents living cells, and F4 represents early-stage apoptotic cells. The apoptosis rates of the three groups are as follows.

Figure 1 shows the apoptosis rate of the three groups of experiments. Among them, the experimental group has the highest content of living cells, which is $91.41 \%$. The viable cells of the control group and the blank group were $86.6 \%$ and $77.7 \%$, respectively, indicating that the experimental group had the largest number of cells that survived under the same conditions. In addition, the amount of apoptosis in the experimental group was also significantly lower than that in the control group. Since the experimental group has the highest number of living cells and a lower apoptosis rate, it indicates that the experimental group has a higher cell survival rate and better biocompatibility. For apoptosis, the early apoptosis rate is used to represent the overall apoptosis of the sample.
As shown in Figure 2, the early apoptosis rate of the experimental group was the lowest at $2.81 \%$, the blank group was $8.17 \%$, and the control group was the highest at $11.9 \%$. The difference between the three groups is obvious. This also shows that the tricalcium phosphate-gelatin composite scaffold material of the experimental group has higher biocompatibility.

4.2. Cytotoxicity Test Results. The cytotoxicity of bone marrow mesenchymal stem cells in experimental group $E$, control group $C$, and blank group $N$ was tested, and the cell proliferation activity (RGR) was calculated. The results are as follows.

As shown in Figure 3, the proliferation activity of bone marrow mesenchymal stem cells in the experimental group, control group, and blank group showed significant differences. The cell proliferation activity of the tricalcium phosphate-gelatin composite scaffold in the experimental group was the highest, at 0.83 at $120 \mathrm{~min}$, the cell proliferation activity of the collagen material in the control group was 0.79 at $120 \mathrm{~min}$, and the blank group was the lowest. This indicates that the tricalcium phosphate-gelatin composite scaffold material is more suitable for cell adhesion and proliferation.

\subsection{Hemocompatibility Evaluation Results}

4.3.1. Coagulation Performance. The greater the absorbance value, the more free hemoglobin in the solution, and the better the anticoagulant performance. Calcium chloride is added to the blood to start the blood coagulation mechanism, and the blood compatibility of the material is evaluated by observing the dynamic coagulation time in vitro. In vitro dynamic coagulation test results of tricalcium phosphate-gelatin composite scaffold material and collagen material are as follows.

As shown in Figure 4, with the increase of time, the absorbance of the two groups of materials showed an overall downward trend, indicating that the coagulation mechanism was activated by sodium chloride, and the content of thrombus began to increase. In the first 45 minutes, the rate of decline is relatively fast, which shows that the degree of activation of coagulation factors is relatively high. However, as time continues to increase, between $60 \mathrm{~min}$ and $120 \mathrm{~min}$, the rate of decline continues to slow down, showing a certain degree of anticoagulant performance. Finally, in the control group with high absorbance, the collagen material has better anticoagulant performance at $68.8 \%$, while the tricalcium phosphate-gelatin composite scaffold material may have a certain cytotoxicity during the crosslinking process, which is related to the phosphoric acid on the cell membrane surface. The combination of bilayers aggravates blood coagulation, and its anticoagulant performance is $64.8 \%$.

4.3.2. Hemolysis Rate. The greater the value of the hemolysis rate is, the more serious the red blood cells will be destroyed. The state stipulates that the hemolysis rate must be less than 


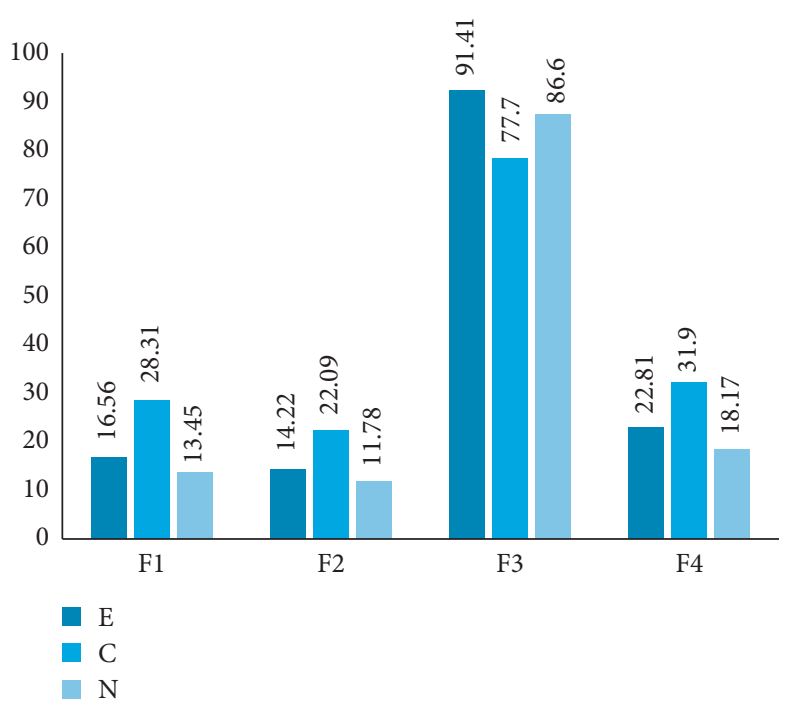

Figure 1: Comparison of cell apoptosis rate.

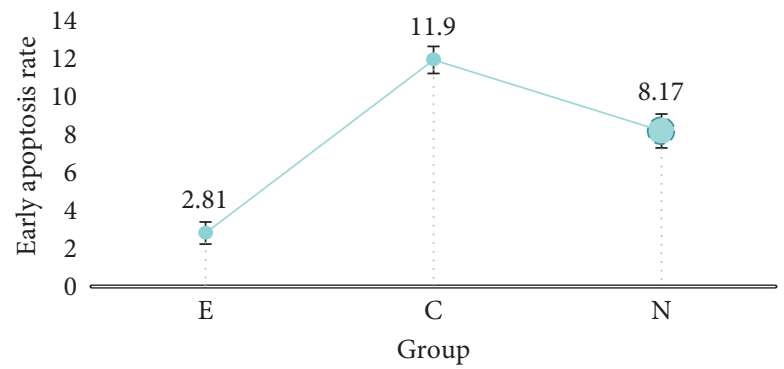

FIGURE 2: Early apoptosis rate of different groups.

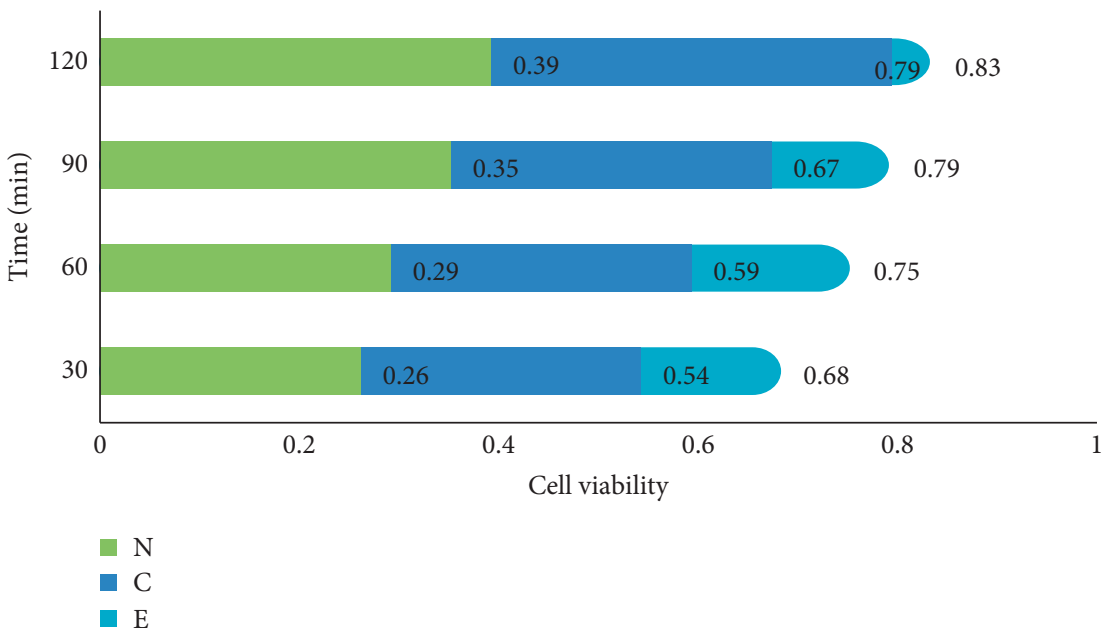

Figure 3: Stem cell proliferation activity detection.

5\%. The material hemolysis rate of experimental group $E$, control group $C$, negative control group $C 1$, and blank group $N$ was tested and compared. The results are as follows.

As shown in Table 1, there were significant differences in absorbance among the four groups, with an average of $0.0566,0.0572$, 0.0516, and 0.2248, respectively. The hemolysis rate of tricalcium phosphate-gelatin composite scaffold was $0.28 \%$ in the experimental group and $0.33 \%$ in the control group. Both of them are lower than the national standard of $5 \%$, and the hemolysis rate of composite materials in the experimental group is lower, and the damage degree of red blood cells is also lower. 


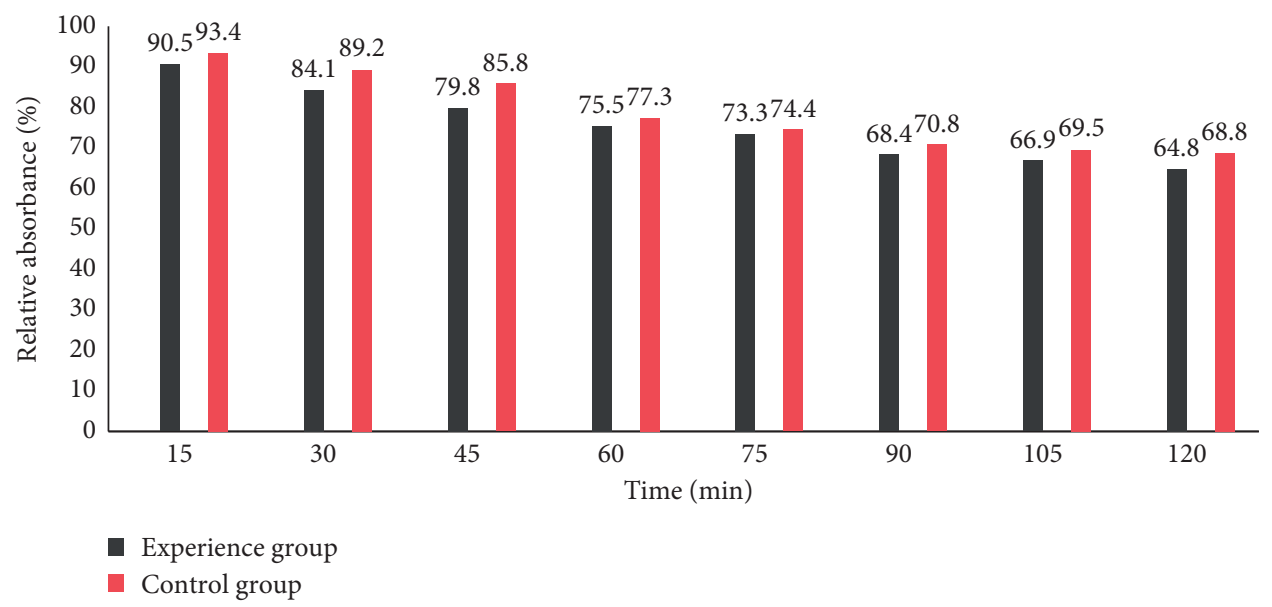

FIgURE 4: In vitro dynamic coagulation test results of the two groups.

TABle 1: Red blood cell hemolysis rate of different groups.

\begin{tabular}{lcccc}
\hline Group & & \multicolumn{2}{c}{ Absorbance } & Average value \\
\hline$E$ & 1 & 2 & 3 & 0.0566 \\
$C$ & 0.0541 & 0.0569 & 0.0587 & 0.0572 \\
$C_{1}$ & 0.0548 & 0.0570 & 0.0599 & 0.0516 \\
$N$ & 0.0511 & 0.0523 & 0.0515 & $0.28 \%$ \\
\hline
\end{tabular}

\section{Conclusions}

Composite biomaterials have been gradually used in the process of sports injury recovery, but the use of this material must consider the compatibility with the individual injury site. This paper studies the biocompatibility of composite biomaterials used to repair sports injuries. First, the rat bone marrow mesenchymal stem cells are cultured to prepare a cell suspension. Then, the tricalcium phosphate-gelatin composite scaffold material and collagen material are scraped into powder to make material extracts, which are the experimental group and the control group, respectively. Apoptosis detection kit and flow cytometer detect the apoptosis rate and survival rate of all samples, and CKK-8 kit detects cytotoxicity and calculates cell proliferation activity. Read the absorbance value with a microplate reader to calculate the coagulation performance and hemolysis rate of different groups. The biocomposite of tricalcium phosphate-gelatin has a good effect on injury recovery and also has high compatibility. Since there are many factors affecting biocompatibility, how to solve the cytotoxicity of composite biomaterials during the crosslinking process is the next problem to be solved in this research. The disadvantage of this study is that this article only conducted preliminary tests on the biocompatibility of the tricalcium phosphate-gelatin composite scaffold, but the long-term biological function needs more experiments to test.

\section{Data Availability}

The data used to support the findings of this study are available from the corresponding author upon reasonable request.

\section{Conflicts of Interest}

The author declares no conflicts of interest with respect to the research, authorship, and/or publication of this article.

\section{References}

[1] K. W. Young, K. C. Park, J. S. Hwang, and H. S. Lee, "Peroneal Tendon repair in sports injury," Journal of Korean Foot and Ankle Society, vol. 23, no. 3, p. 100, 2019.

[2] C. Wang, J. Liu, Y. Liu, B Qin, and D He, "Study on osteogenesis of zinc-loaded carbon nanotubes/chitosan composite biomaterials in rat skull defects," Journal of Materials Science. Materials in Medicine, vol. 31, no. 2, pp. 15-10, 2020.

[3] A. J. Scillia, T. P. Pierce, E. Simone, R. C. Novak, and B. A. Emblom, "Mini-open Incision sports hernia repair: a surgical technique for Core muscle injury," Arthroscopy Techniques, vol. 6, no. 4, pp. e1281-e1284, 2017.

[4] C. Wang, Y. Wang, H. Meng et al., "Research progress regarding nanohydroxyapatite and its composite biomaterials in bone defect repair," International Journal of Polymeric Materials and Polymeric Biomaterials, vol. 65, no. 12, pp. 601-610, 2016.

[5] D. Pieniak, K. Przystupa, A. Walczak et al., "Hydro-thermal fatigue of polymer matrix composite biomaterials," Materials, vol. 12, no. 22, pp. 3650-3651, 2019.

[6] X. Li, S. Zhang, X. Zhang et al., "Biocompatibility and physicochemical characteristics of poly(-caprolactone)/poly(lactide-co-glycolide)/nano-hydroxyapatite composite scaffolds for bone tissue engineering," Materials \& design, vol. 114, pp. 149-160, 2016.

[7] Y. Gu, J. Fernandez, J. S. Baker, and J. Awrejcewicz, "A special Section on Informatics of medical image application in sports 
injury," Journal of Medical Imaging and Health Informatics, vol. 9, no. 3, pp. 540-542, 2019.

[8] Y. Zhang, W. Ni, and Y. Li, "Effect of siliconizing temperature on microstructure and phase constitution of Mo-MoSi2 functionally graded materials," Ceramics International, vol. 44, no. 10, Article ID 11166, 2018.

[9] J. N. Harr and F. Brody, "Sports hernia repair with adductor tenotomy," Hernia, vol. 21, no. 1, pp. 1-9, 2016.

[10] B. Gao, N. Xu, and P. Xing, "Shock wave induced nanocrystallization during the high current pulsed electron beam process and its effect on mechanical properties," Materials Letters, vol. 237, no. 15, pp. 180-184, 2019.

[11] D. Kaul, J. Kahn, L. Huizing et al., "Mid-term functional Outcome and Return to sports after Proximal Hamstring Tendon repair," International Journal of Sports Medicine, vol. 37, no. 7, pp. 570-576, 2016.

[12] F. Alaee, J. Apostolakos, H. Singh et al., "Lateral clavicle fracture with coracoclavicular ligament injury: a biomechanical study of 4 different repair techniques," Knee Surgery, Sports Traumatology, Arthroscopy, vol. 25, no. 7, pp. 2013-2019, 2017.

[13] S. Akahane, Y. Sakai, T. Ueha et al., "Transcutaneous carbon dioxide application accelerates muscle injury repair in rat models," International Orthopaedics, vol. 41, no. 5, pp. 1007-1015, 2017.

[14] D. Steffen and K. Baar, "Loading to Optimize Patellar Tendon repair after injury," Medicine \& Science in Sports \& Exercise, vol. 52, no. 7S, p. 90, 2020.

[15] Z.-D. Meng, C.-J. Wang, Y.-Q. Zhang, C. Luo, Z.-Y. Wang, and W.-C. Li, "Porous hydroxyapatite/Strontium Oxide composite ceramic preparation and properties of biomaterials," Journal of Biomaterials and Tissue Engineering, vol. 9, no. 6, pp. 783-788, 2019.

[16] B. Koodziejska, A. Kaflak, and J. Kolmas, "Biologically Inspired collagen/Apatite composite biomaterials for potential Use in bone tissue regeneration-a review," Materials, vol. 13, no. 7, p. $1748,2020$.

[17] O. M. Otychenko, "Some physico-mechanical properties of composite biomaterials on the basis of biogenic hydroxyapatite with magnetic additives," Functional Materials, vol. 25, no. 4, pp. 695-701, 2018.

[18] S. Chen, M. K. Hassanzadeh-Aghdam, and R. Ansari, “An analytical model for elastic modulus calculation of $\mathrm{SiC}$ whisker-reinforced hybrid metal matrix nanocomposite containing SiC nanoparticles," Journal of Alloys and Compounds, vol. 767, pp. 632-641, 2018.

[19] T. Yokoi, "The development of novel calcium phosphate-polymer composite biomaterials with macro- to nano-level controlled hierarchical structures," Journal of the Ceramic Society of Japan, vol. 127, no. 10, pp. 715-721, 2019.

[20] I. A. Neacsu, A. P. Serban, A. I. Nicoara, R. Trusca, V. L. Ene, and F. Iordache, "Biomimetic composite scaffold based on naturally Derived biomaterials," Polymers, vol. 12, no. 5, p. 1161, 2020.

[21] "Water-based, surfactant-free cytocompatible nanoparticlemicrogel-composite biomaterials-rational design by laser synthesis, processing into fiber pads and impact on cell proliferation," Nephron Clinical Practice, vol. 18, no. 3-4, pp. 740-746, 2017.

[22] X. Wang, G. Wang, L. Liu, and D. Zhang, "The mechanism of a chitosan-collagen composite film used as biomaterial support for MC3T3-E1 cell differentiation," Scientific Reports, vol. 6, no. 1, Article ID 39322, 2016.
[23] P. P. Özyol, E. Özyol, and F. Karel, "Biocompatibility of intraocular lenses," Türk Oftalmoloji Dergisi, vol. 47, no. 4, pp. 221-225, 2017.

[24] I. Palmer, J. Nelson, W. Schatton, N. J. Dunne, F. J. Buchanan, and S. A. Clarke, "Biocompatibility of calcium phosphate bone cement with optimized mechanical properties," Journal of Biomedical Materials Research Part B: Applied Biomaterials, vol. 104, no. 2, pp. 308-315, 2016.

[25] V. Kalidasan, X. L. Liu, T. S. Herng, Y. Yang, and J. Ding, "Bovine serum Albumin-Conjugated Ferrimagnetic Iron Oxide nanoparticles to enhance the biocompatibility and magnetic Hyperthermia performance," Nano-Micro Letters, vol. 8, no. 1, pp. 80-93, 2016.

[26] K. H. Hussein, K.-M. Park, K.-S. Kang, and H.-M. Woo, "Biocompatibility evaluation of tissue-engineered decellularized scaffolds for biomedical application," Materials Science and Engineering: $C$, vol. 67, pp. 766-778, 2016.

[27] D. Algul, A. Gokce, A. Onal, E. Servet, A. I. Dogan Ekici, and F. G. Yener, "In vitrorelease andIn vivobiocompatibility studies of biomimetic multilayered alginate-chitosan $/ \beta$-TCP scaffold for osteochondral tissue," Journal of Biomaterials Science, Polymer Edition, vol. 27, no. 5, pp. 431-440, 2016.

[28] M. Catauro, R. A. Renella, F. Papale, and S. Vecchio Ciprioti, "Investigation of bioactivity, biocompatibility and thermal behavior of sol-gel silica glass containing a high PEG percentage," Materials Science and Engineering: C, vol. 61, pp. 51-55, 2016. 